\title{
ELABORAÇÃO DE ESCALA DIAGRAMÁTICA PARA FURO-DE-BALA E AVALIAÇÃO DE DOENÇAS FOLIARES EM DOIS SISTEMAS DE PRODUÇÃO DE PESSEGUEIRO ${ }^{1}$
}

\author{
MARCIO ALBERTO CHALLIOL ${ }^{2}$, LOUISE LARISSA MAY-DE MIO $^{3}$, FRANCINE LORENA CUQUEL ${ }^{3}$, \\ LINO BITTENCOURT MONTEIRO ${ }^{3}$, BEATRIZ MONTE SERRAT ${ }^{4}$, ANTÔNIO CARLOS VARGAS MOTTA ${ }^{4}$, \\ PAULO JUSTINIANO RIBEIRO JÚNIOR 5
}

\begin{abstract}
RESUMO - As principais doenças foliares que ocorrem em pessegueiro, em Lapa e Araucária, municípios do Estado do Paraná (Brasil), são a ferrugem (Tranzschelia discolor) e o furo-de-bala (Wilsonomyces carpophilus). A primeira causa desfolha logo após a colheita, e a segunda está sempre presente nos pomares, entretanto seus efeitos não são claramente conhecidos e também faltam metodologias para avaliação dessa doença. O presente trabalho objetivou elaborar escala diagramática para furo-de-bala e comparar incidência e severidade de furo-de-bala e da ferrugem em dois sistemas de manejo: produção integrada e convencional. A escala diagramática para furo de bala foi elaborada no primeiro ano de avaliação, selecionando-se folhas com diferentes níveis de severidade, sendo estabelecidos os níveis mínimo e máximo da doença no campo, e os demais níveis foram interpolados de acordo com a acuidade visual. Para comparar os dois sistemas de produção, foram instaladas duas áreas experimentais em duas regiões produtoras (Lapa e Araucária), comparando-se produção integrada (PI) e produção convencional (PC) em duas safras (2002-2003 e 2003-2004), em pomares comerciais de pessegueiro, cultivar 'Chimarrita'. O tratamento PI foi conduzido seguindo as normas técnicas do sistema de PI para pêssego, e o tratamento PC, seguindo o manejo utilizado pelo produtor em cada área. Para avaliar as doenças, foram quantificadas se a incidência e a severidade do furo-de-bala e da ferrugem em ramos marcados, de outubro a abril, nas duas safras. Além disso, foi determinada a desfolha no período de avaliação. A escala diagramática desenvolvida estabeleceu seis níveis de severidade para furo-de-bala, podendo ser indicada para avaliações de comparação entre sistemas e monitoramento da doença no campo. Em Araucária, a incidência e a severidade de furo-de-bala tiveram comportamento inverso entre os anos, sendo menor na PI em primeira safra e maior na segunda, a ferrugem foi menos severa e provocou menor desfolha na PI, no primeiro ano. Em Lapa, não foram observadas diferenças entre os sistemas para furo-de-bala; e para ferrugem, a produção convencional foi melhor, não sendo indicado reduzir pulverizações nesta fase em áreas com alto inóculo do patógeno.
\end{abstract}

Termos para Indexação: Prunus persica, Wilsonomyces carpophilus, Tranzschelia discolor, produção integrada, ferrugem.

\section{SHOT HOLE DIAGRAMMATIC SCALE DEVELOPMENT AND LEAF DISEASES ASSESSMENT IN TWO PRODUCTION SYSTEMS OF PEACH}

\begin{abstract}
The main leaf diseases on peach in Araucaria and Lapa counties, both in the state of Parana, Brazil, are rust (Tranzschelia discolor) and shot hole (Wilsonomyces carpophilus). The first one causes defoliation just after the harvesting, and the second one is always present in the orchards, though its effects are not well known due to the lack of assessment methodology for that disease. This research aimed to develop a diagrammatic scale for shot hole and compare the incidence and severity of leaf diseases in integrated (PI) and conventional (PC) management systems. The shot hole diagrammatic scale was developed in the first year of assessment by selecting leaves with different levels of severity; the minimum and maximum levels of the disease in the fields were established and the other levels were intercalated according to visual accuracy. In order to compare the two production systems - PI and PC, two experimental sites were set in two producing areas (Araucaria and Lapa) with three-year-old commercial peach orchards, cultivar 'Chimarrita', which were evaluated during the 2002/3 and 2003/4 seasons. The PI system was carried out according to techniques recommended for peach PI and the PC was carried out as the system applied by the growers in each area. In order to evaluate the diseases, the incidence and severity of shot hole and rust were quantified in marked branches, from October to April, in two seasons. In addition, defoliation was also determined during the evaluation period. The designed diagrammatic scale established six levels of shot hole severity and can be recommended for the assessment to compare production systems and to monitor the disease in the field. In Araucaria the incidence and severity of shot hole presented inverse behavior between the years, and was lower in the PI system in the first season and higher in the second, whereas rust was less severe and caused less defoliation in the PI system in the first year. In Lapa no differences were observed between the systems in relation to shot hole; as for rust, the conventional management was better. Therefore it is not recommended to reduce pulverization in areas where there is high inoculum of the pathogen.
\end{abstract} Index Terms: Prunus persica, Wilsonomyces carpophilus, Tranzschelia discolor, integrated management, rust.

\section{INTRODUÇÃO}

A produção integrada (PI) de frutas é um sistema que normaliza o cultivo, desde o plantio até a pós-colheita, permitindo introduzir tecnologias que auxiliem na produção de alimentos seguros para o consumidor com menor impacto ao meio ambiente. O PI emprega técnicas físicas, mecânicas, biológicas, culturais e educacionais para controlar o nível de pragas e doenças, a fim de que haja o menor índice de aplicação de pesticidas e baixos níveis de resíduos nos frutos. A produção integrada de pêssego (PIP) no Brasil é regulamentada pelas Normas da PIP (Normas da Produção Integrada de Pêssego, 2003).

Tradicionalmente, o manejo de doenças apresenta dificuldades no sistema de produção integrada, principalmente por

\footnotetext{
${ }^{1}$ (Trabalho 115-2005). Recebido: 27-07-2005. Aceito para publicação:06-10-2006. Trabalho de Iniciação científica do primeiro autor com financiamento do $\mathrm{MAPA} / \mathrm{CNPq}$.

${ }^{2}$ Graduando do curso de Agronomia da Universidade Federal do Paraná, Rua dos Funcionários, 1540, Juvevê, 80035-050 Curitiba-PR; E-mail: marciochalliol@yahoo.com.br

${ }^{3}$ Depto. de Fitotecnia e Fitossanitarismo, SCA/UFPR. Rua dos Funcionários, 1540, 80035-050 Curitiba-PR; Email: maydemio@ufpr.br e lbmonteiro@terra.com.bre francine@ufpr.br

${ }^{4}$ Depto. de Solos e Engenharia Agrícola, SCA/UFPR. Rua dos Funcionários, 1540, Juvevê, 80035-050 Curitiba-PR; Email: bmserrat@ufpr.br e acvmotta@ufpr.br

${ }^{5}$ Depto. de Estatística, SCE/ UFPR, Caixa Postal 19081, CEP 81531-990 Curitiba-PR. Email: prjusti@ufpr.br
} 
ser esta uma tecnologia ainda em desenvolvimento no Brasil (Farias et al., 2003; May-De Mio et al., 2004 [b]). Na maioria dos pomares de pêssego, o controle convencional de patógenos é inadequado, sendo utilizados fungicidas de amplo espectro em sistema de calendário fixo. Além disso, muitas vezes não são consideradas práticas de manejo para redução de fontes de inóculo. Dentre as doenças foliares do pessegueiro, a ferrugem (Tranzschelia discolor (Fuckel) Tranzschel \& Litv.), seguida pelo furo-de-bala (Wilsonomyces carpophilus (Lév.) Adaskaveg, Ogawa \& Butler) são as principais doenças em Lapa e Araucária, no Estado do Paraná (May-De Mio et al., 2004 [a]).

A ferrugem causa prejuízos decorrentes da queda prematura das folhas, nos meses de dezembro a fevereiro, o que estimula a planta a emitir uma brotação nova fora da época. Esse gasto desnecessário de energia por parte da planta resulta na diminuição da produção da safra seguinte (Margarido, 1988; Martins, 1999). O furo-de-bala tem um comportamento diferente, pois, nas condições do Paraná, e em especial para a cultivar 'Chimarrita', não tem sido relatada desfolha na planta (May-De Mio et al., 2004 [a]; Challiol et al., 2004) e, também, é rara a ocorrência de sintomas no fruto; nas folhas, os sintomas aparecem normalmente a partir do final de agosto, atingindo o máximo da doença no mês de outubro, a partir daí ocorre a estabilização a doença. O prejuízo causado por esta doença ainda não é claramente conhecido nesta variedade, pois são raros os trabalhos de avaliação desta doença no campo, faltando também padronização de uso de metodologias. O comportamento destas doenças, na produção integrada comparada com a convencional, por meio de metodologia científica para avaliação, é fundamental para verificar o efeito de mudanças de tecnologias, bem como para orientar futuros trabalhos com as referidas doenças.

O presente trabalho objetivou elaborar escala diagramática para furo-de-bala no pessegueiro e comparar incidência e severidade de furo-de-bala e da ferrugem em dois sistemas de manejo desta fruteira: produção integrada e convencional, no sul do Paraná.

\section{MATERIAL E MÉTODOS}

\section{Descrição da área experimental}

A avaliação de doenças foliares em dois sistemas de produção (Integrada - PI e Convencional - PC) foi realizada nas safras 20022003 e 2003-2004, em pomares comerciais de pessegueiro 'Chimarrita', localizados nos municípios Lapa e Araucária, região sul do Paraná e região metropolitana de Curitiba (PR), respectivamente.

$\mathrm{O}$ pomar experimental em Lapa estava situado à latitude $25^{\circ}$ $46^{\prime} \mathrm{S}$, longitude $49^{\circ} 43^{\prime} \mathrm{W}$, tendo altitude média de 907 metros. Possuía uma área de aproximadamente três hectares, e foi dividida em duas subparcelas: uma conduzida de acordo com as normas da produção integrada de pêssegos, e a outra, de acordo com o modelo convencional. Cada subparcela continha nove linhas de plantio com 65 plantas, onde foram marcadas 10 repetições, de 14 plantas cada, nas cinco linhas de plantio centrais, deixando duas linhas de bordadura de cada lado. A quinta planta de cada repetição foi considerada para avaliação de doenças foliares. Em Araucária, a área experimental situou-se à latitude $25^{\circ} 35^{\prime} \mathrm{S}$, longitude $49^{\circ} 24^{\prime} \mathrm{W}$ e com altitude média de 897 metros. A área do experimento foi de aproximadamente um hectare e foi dividida em quatro linhas de 33 plantas para cada um dos sistemas de produção, sendo consideradas para avaliação as duas linhas centrais. Para cada sistema (PI e PC), foram marcadas quatro repetições de sete plantas cada, sendo avaliadas a terceira e a sexta plantas.

\footnotetext{
- Instituto Tecnológico Simepar - consulta a dados históricos da sua rede de estações meteorológicas. Disponível em: <http://www.simepar.br/>. Acesso em: 10 mar. 2005.
}

\section{Caracterização dos sistemas de manejo}

Os sistemas PIF e PC foram definidos conforme segue: a) Sistema de produção convencional. O manejo e as práticas culturais são definidos pelo produtor em função de experiência própria. Assim, os tratamentos fitossanitários são realizados de acordo com um calendário fixo, sem conhecimento mais preciso da presença de pragas e de patógenos. O solo é mantido sem vegetação na projeção da copa, com uso intensivo de herbicida, e a poda é feita unicamente no inverno.

b) Sistema de produção integrada. Definido de acordo com as Normas para Produção Integrada de Pêssegos (2001 e 2003). As táticas de manejo adotadas foram práticas de cultivo mínimo do solo (roçada na entrelinha e, na linha, no máximo duas aplicações de herbicida por ciclo vegetativo), poda verde e monitoramento de pragas e doenças. Além disso, foram realizados adubação baseada em análises foliar e do solo, cobertura verde na entrelinha, minimização do uso de fungicidas e inseticidas (escolha de produtos mais específicos e fases de aplicação), atualização do produtor nas tecnologias da PIP e registro de todas as atividades executadas no pomar em caderneta de campo.

As pulverizações foram feitas de julho a outubro para furode-bala e em pós-colheita (de dezembro a março) para ferrugem, nas duas safras (Tabela 1), excluindo-se os tratamentos de inverno (realizados com calda bordaleza, para ambos) e os tratamentos de florada e pré-colheita, específicos para podridão-parda.

Os dados climáticos do município de Lapa foram obtidos do SIMEPAR ${ }^{1}$ e do município de Araucária foram da Secretaria Municipal da Agricultura.

\section{Elaboração da escala diagramática para furo-de-bala}

A escala diagramática da severidade de W. carpophilus foi elaborada neste trabalho de acordo com a metodologia de Horsfall \& Barrat (1945) e Nutter \& Schultz (1995), seguindo modelos logísticos, na qual se estabelecem níveis de severidade respeitando as limitações da acuidade visual humana. Assim, na elaboração da escala, foram coletadas 300 folhas no campo, em outubro de 2002 (período de maior severidade da doença nesta região) e determinados padrões de máximo e mínimo de doenças com o uso de medidor de área foliar WINRIZO $^{\circledR}$. Para o desenho da escala foram considerados o formato de uma folha-padrão e a distribuição das lesões no limbo foliar em cada nível, seguindo o padrão da doença no campo.

Após o estabelecimento dos níveis máximo e mínimo de severidade da doença, os níveis intermediários da escala foram determinados matematicamente, seguindo incrementos logarítmicos. Uma vez definidas as percentagens de doença a serem representadas na escala, calculou-se a área média padrão de uma folha de pessegueiro, sobre a qual foram marcadas as lesões provocadas pelo fungo, de modo que estas retratassem os sintomas observados no campo.

\section{Metodologia de avaliação das doenças foliares}

Para a avaliação de furo-de-bala e ferrugem, foram selecionados dois ramos por árvore marcada em Araucária e quatro ramos por árvore em Lapa. O ramo avaliado foi um "ramo do ano", de tamanho médio e localizado na altura mediana da planta, no sentido da entrelinha.

A intensidade de cada doença do pessegueiro foi avaliada de outubro a abril de cada safra, sob dois aspectos: incidência e severidade. Para avaliar a incidência, considerou-se a relação entre o número de folhas do ramo marcado as quais apresentavam qualquer sintoma e o número total de folhas do ramo. Para avaliar severidade da ferrugem, utilizou-se escala desenvolvida por Martins (1994), com 10 níveis de severidade, 1 a 10 correspondendo a 0,$06 ; 0,09 ; 0,19$; 0,$28 ; 0,50 ; 0,85 ; 1,48 ; 2,52 ; 4,22$ e $6,97 \%$ da área foliar afetada pela doença. Para avaliar a severidade de furo-de-bala, utilizou-se escala descrita no item anterior. 

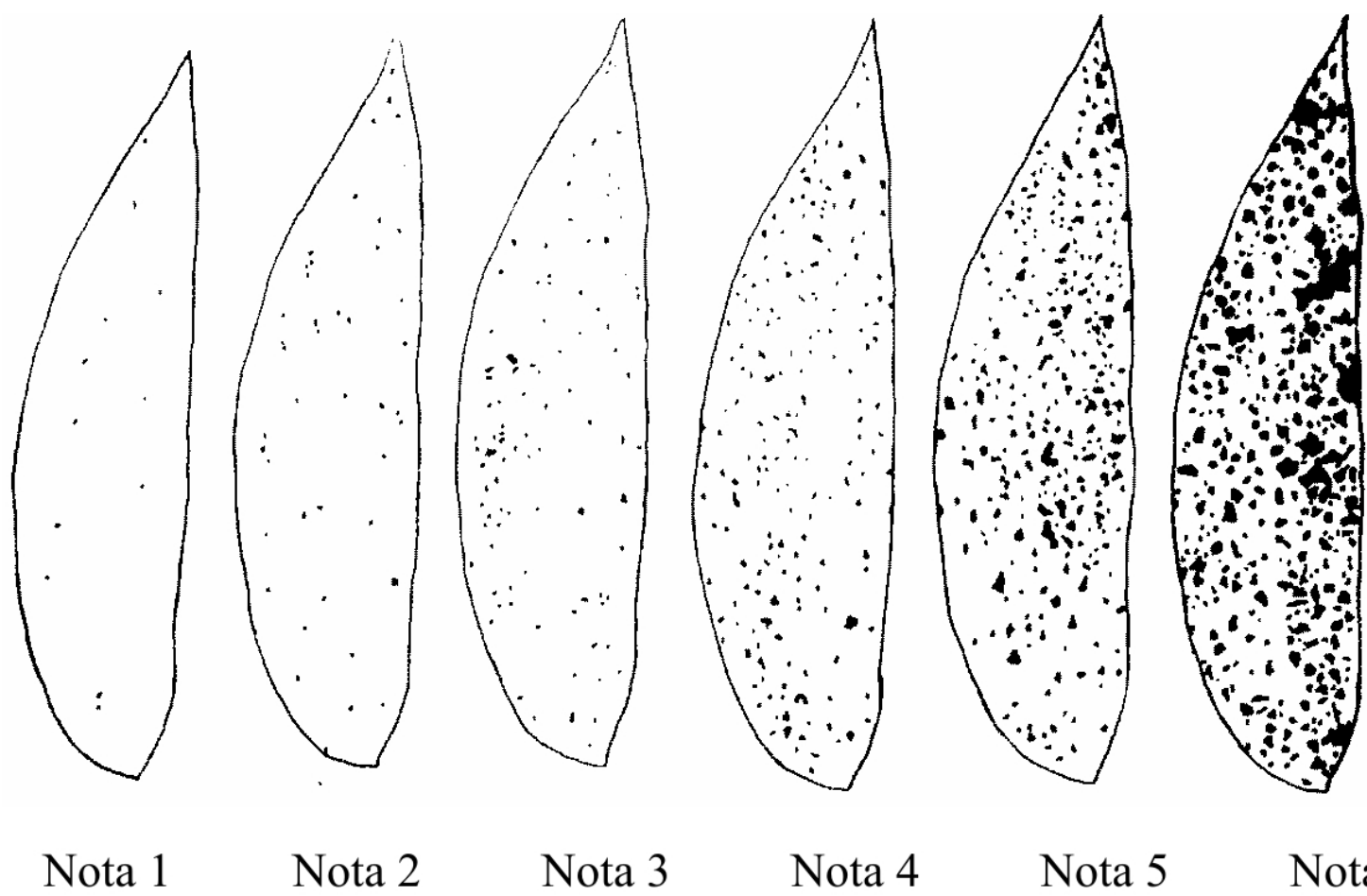

Nota 2

Nota 3

Nota 4

\section{Nota 5}

Nota 6

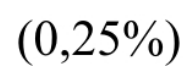

$(0,66 \%)$

$(1,71 \%)$

$(4,39 \%)$

$(10,80 \%)$

FIGURA 1 - Escala diagramática do Furo-de-Bala em pessegueiro. Notas representam a percentagem de sintomas da doença (valores entre parênteses) e também perfurações que se formam pelo destacamento do tecido afetado por W. carpophilus. Elaboração a partir do modelo de Horsfall \& Barrat (1945) e Nutter \& Schultz (1995).

TABELA 1 - Número e tipo de fungicidas aplicados na cultura de pessegueiro, em experimento para comparação dos dois sistemas de produção integrada (PI) e convencional (PC) para controle de furo-de-bala e de ferrugem.

\begin{tabular}{|c|c|c|}
\hline \multirow{2}{*}{$\begin{array}{c}\text { Local / Safra / } \\
\text { Sistema }\end{array}$} & \multicolumn{2}{|c|}{ Número e tipo de fungicidas aplicados em duas fases } \\
\hline & Antes colheita ${ }^{1}$ & Após colheita ${ }^{2}$ \\
\hline
\end{tabular}

\section{LAPA-PR}

\begin{tabular}{ll}
\hline Safra 2002 - PI & 1 captana, 1 mancobebe, 1 captana + fosfito de K, \\
2003 & 1 captana (4).
\end{tabular}

1 mancozebe, 1 tebuconazole (2)

PC

3 mancozebe (3)

Safra 2003 - PI 2004
1 captana, 1 mancozebe + fosfito de CaB, 1 captana,

1 calda sulfocálcica, 1 captana (5).

PC 1 calda sulfocálcica, 1 captana, 1 mancozebe, 2 calda sulfocálcica, 1 mancozebe, 1 calda sulfocálcica, 1 mancozebe $(8)$
2 calda sulfocálcica (2).

1 mancozebe, 1 calda sulfocálcica, 1 mancozebe, 1 calda sulfocálcica (4).

\section{ARAUCÁRIA-PR}

\begin{tabular}{|c|c|c|}
\hline \multirow[t]{2}{*}{$\begin{array}{l}\text { Safra } 2002-\mathrm{PI} \\
2003\end{array}$} & $\begin{array}{l}1 \text { captana, } 1 \text { mancozebe, } 1 \text { captana }+ \text { fosfito } K, 1 \text { captana, } \\
1 \text { captana }+ \text { fosfito } K(5) .\end{array}$ & 2 tebuconazole (2). \\
\hline & $\begin{array}{l}1 \text { benomyl, } 1 \text { captana, } 2 \text { mancozebe, } 1 \text { oxicloreto de cobre, } 2 \\
\text { mancozebe, } 1 \text { captana }(8) .\end{array}$ & 2 mancozebe (2). \\
\hline \multirow[t]{2}{*}{$\begin{array}{l}\text { Safra } 2003 \\
2004\end{array}$} & 1 captana, 1 mancozebe, 4 captana (6). & 1 tebuconazole 1 mancozebe $(2)$. \\
\hline & $\begin{array}{l}1 \text { benomyl, } 1 \text { captana, } 1 \text { mancozebe, } 1 \text { benomyl, } 1 \text { mancozebe, } \\
1 \text { benomyl, } 2 \text { captana, } 1 \text { enxofre (9). }\end{array}$ & 1 tebuconazole, 1 mancozebe (2). \\
\hline
\end{tabular}

\footnotetext{
*As dosagens dos produtos seguiram recomendação do fabricante.

${ }^{1}$ Aplicações que têm influência em furo-de-bala.

${ }^{2}$ Tratamentos para controle da ferrugem.

Valores entre parêntese indicam o total de pulverizações antes ou após a colheita.
} 
Outro parâmetro avaliado foi a desfolha decorrente da ferrugem. A avaliação foi realizada no mesmo ramo utilizado para avaliar as doenças, sendo considerado o número de folhas por ramo marcado no enfolhamento máximo, em relação ao número de folhas no final das avaliações. A diferença, em percentual, era a desfolha.

\section{Análise estatística dos dados}

Para incidência e severidade, os dados das datas de avaliação foram integralizados no tempo e transformados em área abaixo da curva de progresso da doença (AACPD) pelo método da integração trapezoidal (Berger, 1988). A análise estatística da AACPD foi baseada no ajuste dos dados segundo o modelo linear generalizado (McCullagh \& Nelder, 1989) para cada uma das variáveis analisadas. Corrigiu-se para o delineamento e coletas ao longo do tempo, e incluiuse, ao final do modelo, um termo para o tipo de plantio, cuja significância foi avaliada pelo teste da razão de verossimilhança. As análises foram realizadas utilizando o sistema estatístico R ( $\mathrm{R}$ Development Core Team, 2005).

\section{RESULTADOS E DISCUSSÃO}

\section{Escala diagramática desenvolvida}

Com o resultado da elaboração da escala do furo-de-bala foram definidos seis níveis de doença, com notas de um a seis, correspondendo a 0,$25 ; 0,66 ; 1,71 ; 4,39 ; 10,8$ e 24,23\% da área foliar afetada (Figura 1). Shaw et al. (1990) elaboraram uma escala para esta doença, com cinco níveis de severidade para a cultura da ameixa, correspondendo o menor nível a $1 \%$ e o maior a mais de $20 \%$ de severidade. Apesar de os valores estarem próximos aos da escala desenvolvida neste trabalho, aquela não seguiu padrões de acuidade visual, além de não estabelecer o limite máximo da doença. Com uso da escala diagramática desenvolvida neste trabalho, foi possível quantificar a severidade entre os dois sistemas de manejo em Araucária e Lapa (Tabela 2), podendo ser indicado o uso desta escala para monitoramento nas áreas com PI de pessegueiro.

Incidência e severidade do furo-de-bala e da ferrugem ao longo do tempo, nos dois sistemas e áreas experimentais

Em relação à incidência do furo-de-bala na safra 2003-2004, em Araucária, a doença foi pouco severa, variando entre 40 a 50\%, enquanto na safra 2002-2003 chegou a $90 \%$ das folhas com doença. A severidade atingiu nota 1,43 para PI e 2,73 para PC ao final da safra 2002-2003, o que possibilitou diferenciar os dois sistemas de produção.

As AACPD da incidência e da severidade do furo-de-bala em Araucária foram 37 e 71\%, respectivamente, maiores no sistema de PC comparada a PI na safra 2002-2003 (Tabela 2). Já safra 20032004, ocorreu o inverso, o PC foi superior a PI (incidência e severidade $16 \%$ maior no PI), provavelmente em função das aplicações do fungicida de ação sistêmica benomyl, que conferiu melhor proteção plantas que a captana aplicada no sistema de PI. Apesar da eficiência do produto benomyl, ele não é recomendado na produção integrada em função do seu modo de ação específico, que tem contribuído para seleção de isolados resistentes de vários fungos (Zehr, 1982; May-De Mio et al., 2004 [b]). No entanto, deve-se considerar que, na segunda safra, em ambos os sistemas e áreas experimentais, a doença manteve-se com baixa severidade, chegando ao máximo de nota 2 na escala diagramática de furo-de-bala, o que corresponde em torno de $0,6 \%$ de área foliar afetada pela doença, também evidenciado pelos valores menores de AACPD de 23 e 27 para PC e PI, respectivamente.

Em Lapa, a incidência e a severidade de furo de bala foram similares $(p>=0,05)$ entre os sistemas de produção para as duas safras (Tabela 2). Mesmo com reduções de 71 a $57 \%$ do número de fungicidas aplicados, na primeira e segunda safras, respectivamente, a PI foi equivalente a PC.

TABELA 2 - Desfolha e área abaixo da curva do progresso das doenças (AACPD) furo-de-bala e ferrugem, para variável incidência e severidade, durante as safras de 2002-2003 e 2003-2004 nos sistemas de produção integrada (PI) e produção convencional (PC), nas duas áreas experimentais, Araucária e Lapa - PR.

\begin{tabular}{|c|c|c|c|c|c|c|}
\hline \multirow{2}{*}{\multicolumn{2}{|c|}{$\begin{array}{c}\text { Local e } \\
\text { Sistema de produção }\end{array}$}} & \multicolumn{2}{|c|}{ Furo-de-bala (em AACPD) ${ }^{1}$} & \multicolumn{2}{|c|}{ Ferrugem (em AACPD) ${ }^{1}$} & \multirow{2}{*}{$\begin{array}{l}\text { Desfolha } \\
(\mathrm{em} \%)\end{array}$} \\
\hline & & Incidência & Severidade & Incidência & Severidade & \\
\hline \multicolumn{7}{|c|}{ ARAUCÁRIA } \\
\hline \multirow{2}{*}{$\begin{array}{l}\text { Safra } \\
2002- \\
2003\end{array}$} & PI & $4534,69^{*}$ & $70,21 *$ & $2429,69^{\mathrm{ns}}$ & $52,50^{*}$ & $20,65^{*}$ \\
\hline & $\mathrm{PC}$ & 6215,93 & 120,14 & 2931,90 & 82,06 & 43,34 \\
\hline \multirow{2}{*}{$\begin{array}{l}\text { Safra } \\
2003- \\
2004\end{array}$} & PI & $4603,58^{*}$ & $27,62 *$ & $1974,18^{*}$ & $71,60^{*}$ & $32,80^{\mathrm{ns}}$ \\
\hline & $\mathrm{PC}$ & 3949,46 & 23,77 & 2337,24 & 81,61 & 31,69 \\
\hline \multicolumn{7}{|c|}{ LAPA } \\
\hline \multirow{2}{*}{$\begin{array}{l}\text { Safra } \\
2002- \\
2003\end{array}$} & PI & $5728,15^{\mathrm{ns}}$ & $(1,26)^{\mathrm{ns}}$ & $2488,76^{*}$ & $(9,50)^{\mathrm{ns}}$ & $10,10^{\mathrm{ns}}$ \\
\hline & $\mathrm{PC}$ & 5315,86 & $(1,39)^{2}$ & 2741,23 & $(9,80)$ & 8,85 \\
\hline \multirow{2}{*}{$\begin{array}{l}\text { Safra } \\
2003- \\
2004\end{array}$} & PI & $3753,65^{\mathrm{ns}}$ & $28,16^{\mathrm{ns}}$ & $3914,61 *$ & $139,70^{*}$ & $46,08^{*}$ \\
\hline & $\mathrm{PC}$ & 3496,27 & 26,93 & 3067,39 & 110,12 & 38,96 \\
\hline
\end{tabular}

\footnotetext{
ns Indica que não houveram diferenças significativas entre os sistemas.

* Indica diferença significativa ao nível de $5 \%$ entre os sistemas, pela análise de deviance.

${ }^{1}$ Os maiores valores representam a maior quantidade de doença (incidência ou severidade).

${ }^{2}$ Os valores entre parênteses são provenientes de uma única avaliação, em dezembro para furo-de-bala e em março, para ferrugem da safra 2002-2003, em Lapa.
} 
Em relação à ferrugem, a PI foi mais eficiente para reduzir a severidade da doença em Araucária para os dois anos avaliados. Entretanto, para desfolha, esta diferença foi observada apenas no primeiro ano, quando a PI apresentou aproximadamente $50 \%$ a menos de desfolha comparada com a PC. Em Lapa, a PI foi superior, avaliandose incidência no primeiro ano e inferior em todas as variáveis (incidência, severidade e desfolha), no segundo ano (Tabela 2). A maior severidade de ferrugem em Lapa para a PI é decorrente de o produtor não ter seguido a recomendação de fungicidas (mancozebe e tebuconazole) após a colheita no ciclo 2003-2004 (Tabela 1).

Em alguns casos, a incidência chega a valores próximos ao máximo em todos os tratamentos, deixando assim de ser uma variável para uso comparativo do desenvolvimento da epidemia. Como exemplo disso, a incidência de ferrugem não diferiu nos sistemas na safra 2002-2003 em Araucária, mas a severidade mostrou que, na PI, a doença foi menos severa (Tabela 2). Neste caso, a severidade tornase uma característica de referência, sendo também considerada de fundamental importância por diversos autores para avaliação de doenças foliares em várias culturas (Shaw et al., 1990; Martins, 1999; Carvalho et al., 2002).

Comparando-se as áreas, o pomar em Lapa apresentou intensidade de ferrugem superior ao final das avaliações em relação à Araucária, provavelmente em decorrência de o inóculo inicial da doença ser maior naquele pomar. Este aumento de inóculo pode ter sido causado pela falha na adoção dos tratamentos preconizados pela produção integrada. Outro fato relevante é que o pomar em Lapa tem aproximadamente 15 hectares de diferentes variedades de pessegueiros e, por muito tempo, ficou sem manejo eficiente para a ferrugem, o que mantém a doença por mais tempo sobrevivendo na área. Para esta doença, segundo estimado na elaboração da escala diagramática, só ocorrerá desfolha a partir de $6 \%$ de severidade, o que equivale à nota 10 da escala de avaliação. Estes dados estão de acordo com o trabalho desenvolvido por Martins (1999). Mas Challiol et al. (2004), avaliando eficiência de fungicidas para controle de ferrugem em pessegueiro, verificaram desfolha de $30 \%$ maior na testemunha em relação a parcelas tratadas com fungicidas, mesmo quando os níveis médios de severidade nas folhas remanescentes no ramo avaliado estavam com nota quatro $(0,28 \%$ de severidade). Tal fato indica que, possivelmente, $30 \%$ das folhas já tinham atingido severidade próxima a $6 \%$ e haviam caído, deixando nesta fase a severidade do ramo mais baixa que em avaliações anteriores.

Os produtos utilizados neste trabalho, na produção integrada e na convencional, mostraram-se eficientes no controle da epidemia de ferrugem, como já relatado por Martins (1994), que testou vários dos produtos utilizados, dentre os quais captana, mancozebe e tebuconazole, nas formulações CE e PM. Da mesma forma, Carvalho et al. (2002) observaram eficiência de mancozebe e tebuconazole combinados a inseticidas, reduzindo a desfolha das plantas. No Paraná, estes produtos demonstraram eficiência com controle de 50 $\%$ com mancozebe e de 67 a $77 \%$ (de acordo com a dosagem) com tebuconazole em relação à testemunha (Challiol et al., 2004).

Para o manejo dessas doenças foliares dentro da PI, o importante é a redução do número de aplicações de fungicidas e demais agroquímicos, como já demonstrado por Fachinello et al. (2003), quando observaram uma redução de $37,5 \%$ no número de aplicações de agroquímicos. Em trabalho realizado por May-De Mio et al. (2004 [b]) no Paraná, na mesma área de estudo, o número de tratamentos totais com agroquímicos em Araucária foi menor na PI: no primeiro ano foram feitas 20 pulverizações no PI contra 30 no PC e, no segundo ano, foram feitas 17 no PI contra 22 no PC. Outra constatação importante no mesmo trabalho é que, com o passar do tempo, os sistemas se nivelam, pois o produtor acaba transferindo para a área da PC as práticas de manejo que resultam em ganhos econômicos que foram utilizados na PI.
As avaliações e o monitoramento das doenças foliares nos sistemas de PI e PC são importantes para comparar os manejos fitossanitários. Ainda que em algumas situações a PC tenha apresentado menor intensidade de doença, deve-se destacar que, no sistema de PI, reduzem-se as pulverizações de fungicidas e se obtém uma fruta de melhor qualidade, do ponto de vista de segurança alimentar.

A princípio, o sistema de produção integrada de frutas não tem por objetivo ser mais produtivo que o modelo convencional, o qual utiliza grande quantidade de fungicidas, muitas vezes de forma indiscriminada, mas, sim, ser o mais próximo deste. Isto só é possível com a utilização racional das diversas formas de controle, utilizandose de ferramentas de monitoramento de doenças e pragas, assim como todas aquelas já citadas anteriormente como práticas corriqueiras desse sistema de produção.

\section{CONCLUSÃO}

1) A escala diagramática desenvolvida estabeleceu seis níveis de severidade para furo-de-bala, podendo ser indicada para avaliações de comparação entre sistemas de produção de pessegueiro e monitoramento da doença no campo.

2) Em Araucária, a incidência e a severidade de furo-de-bala tiveram comportamento inverso entre os anos, sendo menor na PI na primeira safra e maior na segunda; a ferrugem foi menos severa e provocou menor desfolha na PI, no primeiro ano. Em Lapa, não foram observadas diferenças entre os sistemas para furo-de-bala; e para ferrugem, a produção convencional foi melhor, não sendo indicado reduzir pulverizações na PI, nesta fase, em áreas com alto inóculo do patógeno.

\section{AGRADECIMENTOS}

Ao Engenheiro Agrônomo Edir Osmar Buske, proprietário do pomar em Lapa, e ao Sr. Waldomiro Gayer Neto, proprietário do pomar em Araucária, ao Ministério da Agricultura, Pecuária e Abastecimento (MAPA), e ao Conselho Nacional de Apoio à Pesquisa (CNPq), pelo suporte financeiro.

\section{REFERÊNCIAS}

BERGER, R.D. The analysis of the effects of control measures on the development of epidemics. In: KRANZ, J.; ROTEM, J. (Ed.) Experimental techniques in plant disease epidemiology. Heidelberg: Springer-Verlang, 1988. p.137-151.

CARVALHO, V.L.; GONÇALVES-GERVÁSIO, R.C. R.; SANTACECÍLIA, L.V.C.; KATO, C.M.; FOUREAUX, L.V.; CAMPELO, M.G. Alternativas de controle da ferrugem do pessegueiro [Tranzschelia discolor (Feckel) Tranzschel Litivinov]. Ciência Agrotécnica, Lavras, v.26, n.2, p.227-231, 2002.

CHALLIOL, M.A.; MAY-DE MIO, L.L.; MOREIRA, L.M. Avaliação temporal da ferrugem do pessegueiro e eficiência de fungicidas no controle. In: CONGRESSO BRASILEIRO DE FRUTICULTURA, 17., 2004, Florianópolis. Anais... Florianópolis: SBF, 2004. CD ROM.

FACHINELLO, J.C.; TIBOLA, C.S.; VICENZI, M.; PARISOTTO, E.; PICOLOTTO, L.; MATTOS, M.L.T. Produção integrada de pêssegos: três anos de experiência na região de Pelotas - RS. Revista Brasileira de Fruticultura, Jaboticabal, v.25, n.2, p.256258, 2003.

FARIAS, R.M.; NUNES, J.L.S.; MARTINS, C.R.; GUERRA, D.S.; ZANINI, C.; MARODIN, G.A.B. Produção convencional x integrada em pessegueiro cv. Marli na depressão central do Rio Grande do Sul. Revista Brasileira de Fruticultura, Jaboticabal, v.25, n.2, p.253-255, 2003. 
HORSFALL, J.G.; BARRAT, R.W. An improved grading system for measuring plant diseases. Phytopathology, St Paul, v.35, p.655, 1945. Abstract

MARGARIDO, S.M.F. Pêssego e nectarina: belezas e delícias do pomar. São Paulo: Ícone Editora, 1988. p.12-17.

MARTINS, M.C. Quantificação dos parâmetros monolíticos e controle químico da ferrugem do pessegueiro. 1994. 68 f. Tese (Mestrado) - Escola Superior de Agricultura "Luiz de Queiroz", Universidade de São Paulo, Piracicaba, 1994.

MARTINS, M.C. Caracterização Morfofisiológica de Tranzschelia discolor, efeito da umidade na patogênese e controle da ferrugem do pessegueiro. 1999. 81f. Tese (Doutorado) - Escola Superior de Agricultura "Luiz de Queiroz", Universidade de São Paulo, Piracicaba, 1999

MAY-DE MIO, L.L.; GARRIDO L.; UENO, B. Doenças de fruteiras de caroço, In: MONTEIRO, L.B.; MAY-DE MIO, L.L.; SERRAT, B.M.; MOTTA, A.C.V.; CUQUEL, F.L. Fruteiras de caroço: uma visão ecológica. Curitiba, 2004a. cap.10, p.169-222.

MAY-DE MIO, L.L.; MONTEIRO, L.B.; MOTTA, A.C.V.; SERRAT, B.M.; CUQUEL, F.L.; JUSTINIANO Jr., P. Comparação entre os sistemas Convencional e Integrado para produção de pêssegos em Araucária - PR. CONGRESSO BRASILEIRO DE FRUTICULTURA, 17., 2004, Florianópolis. Anais... Florianópolis: SBF, 2004b. CD ROM.
McCULLAGH, P.; NELDER, J.A. Genaralized linear models. $2^{\text {nd }}$ ed. London: Chapman and Hall, 1989.

NORMAS DEPRODUÇÃOINTEGRADADEPESSSEGO(PIP): Versão II. Ed. Pelotas: Ufpel/Embrapa/ Ufrgs/ Urcamp, 2003. 55 p.

NUTTER, Jr., F.W.; SCHULTZ, P.M. Improving the accuracy and precision of disease assessments: selection of methods and use of computer-aided training programs. Canadian Journal of Plant Pathology, Toronto, 17, p.174-184, 1995.

R DEVELOPMENT CORE TEAM. R: A language and environment for statistical Computing. R Foundation for Statistical Computing, Vienna, Austria. ISBN 3-900051-07-0. Disponível em: <http://www.R-project.org>. Acesso em: fev. 2005.

SHAW, D.A.; ADASKAVEG, J.E.; OGAWA, J.M. Influence of Wetness period and temperature on infection and development of shothole disease of almond caused by Wilsonomyces carpophilus. Phytopathology, St Paul, n.80, p.749-756, 1990.

SIMEPAR - Instituto Tecnológico Simepar - consulta a dados históricos da sua rede de estações meteorológicas. Disponível em: <http://www.simepar.br/>. Acesso em: 10 mar. 2005.

ZEHR, E.I. Control of brown rot in peach orchards. Plant Disease, St. Paul, v.66, n.12, p.1.101-1.105, 1982. 